\title{
EFFECTS OF ARSENIC ON DIVERSITY OF RICE FIELD WEEDS
}

\author{
Abdul Aziz and Ashfaque Ahmed \\ Department of Botany, University of Dhaka, Dhaka-1000, Bangladesh
}

Key words: Arsenic, Rice fields, Weeds, Diversity

In Bangladesh use of groundwater for irrigation and drinking is increasing the arsenic contamination allarmingly.(1) Studies in home and abroad confirmed that a substantial amount of this heavy metal is absorbed by plants ${ }^{(2-5)}$ and it affects $\mathrm{PO}_{4}-\mathrm{P}$ absorption $^{(2,5)}$ thereby preventing ATP generation. ${ }^{(5-7)}$ A survey of rice fields at. Sonargaon in Narayanganj district revealed that farmers use arsenic (476 - $497 \mu \mathrm{g} / \mathrm{l})$ contaminated shallow tube well water for irrigation. Attempts were therefore taken to see if the diversity of weeds in rice field irrigated with the arsenic contaminated groundwater is affected.

An experiment was carried out to determine the effect of arsenic on the diversity of rice field weeds at Nilkanda Union under P.S. Sonargaon in Narayanganj district, which lies between $23^{\circ} 32^{\prime}$ and $23^{\circ} 46^{\prime} \mathrm{N}$ and $90^{\circ} 31^{\prime}$ and $90^{\circ} 41 \mathrm{E}^{\prime}$. The study area is situated in the Meghna floodplain in Silmondi and Narailbag soil series. The experiment was conducted in $19 \times 19 \mathrm{~m}$ plots of five replicates arranged in a complete randomized block design. High yield rice variety (BR-28) was grown and irrigated with pond water (as control) and contaminated groundwater from a shallow tube well. The water quality and soil characteristics are described elsewhere.(8) Recommended doses of NPK fertilizers were applied to all plots.

The weed vegetation in the rice plots was studied after two months of transplantation. The important vegetational attributes which may be measured readily are size, number and distribution of component parts. The use of small, square areas (quadrat) of known size as the basic study unit for the investigation of these attributes has been recommended. $.^{(9,10)} \mathrm{A} 1 \times 1 \mathrm{~m}$ wooden quadrat was used in the present study. A total of five quadrats were randomly placed in each plot. Number of plants fall in each quadrat were listed and counted. From the data the phytosociological analysis was done. Besides, Shannon-Weaver ${ }^{(11)}$ and Simpson ${ }^{(12)}$ indices of diversity were determined.

A total of 14 weeds were recorded from the experimental plots, a list of which and their presence or absence in the control and treatment plots are given in Table 1. It was revealed that the control plot showed higher species diversity (11 taxa) than the plots irrigated with arsenic contaminated water (eight taxa). 
Phytosociological analysis and various indices were determined from the data of five quadrats of a plot which are shown in Table 2. Maximum importance value index (IVI) was recorded 50.04 in Lindernia antipoda and Eriocolon sp. and minimum was 17.29 in Hediotis corymbosa in the control. In the treatment Lindernia antipoda had maximum IVI (70.46) followed by Eriocolon sp. and Panicum sp., whereas a minimum value of 19.26 was found in C. rotundus, i.e. both the control and treatment were dominated by Lindernia antipoda and Eriocolon sp. Arsenic appears to enhance growth of Cyperus rotundus, Eclipta alba and Fimbristilis sp., while Cynodon dactylon, Echinochloa colonum, Enhydra flactuans and Lippia nodiflora are sensitive to arsenic (Table 2). Arsenic has been found to increase the growth and yield of citrus plants at low concentration (below $20 \mathrm{mg} / \mathrm{kg}$ dry soil, an upper permissible limit) of arsenic in the soil.(13) The study area had $2.5-8.0 \mathrm{mg} / \mathrm{kg}$ dry soil. Plants tolerant to large arsenic applications $(670 \mathrm{ppm})$ to soils have been reported.(14) Genotypic variation in tolerance to arsenic toxicity among Brassica juncea L. genotype have been observed. ${ }^{(15)}$ The sensitivity of some weeds to even low concentration of soil arsenic may be due to competitive uptake of arsenic to phosphorus by these plants, thereby prevents ATP generation.(5-7) Marked yield reduction of sweet corn occurred at 5 ppm of extractable arsenic. ${ }^{(16)}$

Table 1. List of weeds present in plots irrigated with pond water $(9.50 \pm 0.50 \mu \mathrm{g} / \mathrm{l}$ arsenic) and arsenic contaminated $(476 \pm 3 \mu \mathrm{g} / \mathrm{l})$ water from a shallow tube well. Presence $(+)$ or absence (-) are also shown.

\begin{tabular}{lcc}
\hline Name of the weeds & Pond water & Shallow tube-well water \\
\hline Alternanthera sessilis & + & - \\
Cynodon dactylon & + & - \\
Cyperus exceltatum & + & + \\
C. rotundus & - & + \\
Eclipta alba & - & + \\
Echinochloa colonum & + & - \\
Enhydra flactuans & + & - \\
Eriocaulon setaceum. & + & + \\
Fimbristilis sp. & - & + \\
Hediotis corymbosa & + & - \\
Hydrocotyle rotundifolia & + & + \\
Lindernia antipoda & + & + \\
Lippia nodiflora & + & - \\
Panicum sp. & + & + \\
\hline
\end{tabular}

Importance value index indicated that Cynodon dactylon, Echinochloa colonum, Enhydra flactuans and Lippia nodiflora are very sensitive to arsenic; Lindernia antipoda and Eriocaulon setaceum were not affected at all, while growth of Cyperus rotundus, Eclipta alba and Fimbristilis sp. were enhanced in presence of arsenic. 
It was revealed that the control (irrigated with pond water) showed higher species diversity than the treatment (irrigated with arsenic contaminated water). It was suggested that because of the so called soil/plant barrier effect, elevated arsenic concentrations in soil may well reduce crop production substantially before enhanced food chain accumulation occurred.(17)

Table 2. Phytosociological analysis, Shannon index (H) and Simpson's index (D) of diversity of weeds in plots irrigated with pond water and Shallow tube well water (arsenic contaminated).

\begin{tabular}{|c|c|c|c|c|c|c|c|c|c|}
\hline $\begin{array}{l}\text { Shallow tube } \\
\text { well water }\end{array}$ & Density & $\begin{array}{l}\text { Fre- } \\
\text { quency }\end{array}$ & $\begin{array}{l}\text { Abun- } \\
\text { dance }\end{array}$ & $\begin{array}{c}\text { Relative } \\
\text { density }\end{array}$ & $\begin{array}{l}\text { Relative } \\
\text { frequency }\end{array}$ & $\begin{array}{l}\text { Relative } \\
\text { abundance }\end{array}$ & IVI & $\mathrm{H}$ & $\mathrm{D}$ \\
\hline $\begin{array}{l}\text { Alternanthera } \\
\text { sessilis }\end{array}$ & $\begin{array}{l}0.2 \\
(-)\end{array}$ & $\begin{array}{l}0.2 \\
(-)\end{array}$ & $\begin{array}{l}1.0 \\
(-)\end{array}$ & $\begin{array}{c}1.96 \\
(-)\end{array}$ & $\begin{array}{c}3.86 \\
(-)\end{array}$ & $\begin{array}{c}4.76 \\
(-)\end{array}$ & $\begin{array}{c}10.57 \\
(-)\end{array}$ & \multirow{14}{*}{$\begin{array}{c}3.135 \\
(2.751)\end{array}$} & \multirow{14}{*}{$\begin{array}{c}0.865 \\
(0.836)\end{array}$} \\
\hline $\begin{array}{l}\text { Cynodon } \\
\text { dactylon }\end{array}$ & $\begin{array}{l}0.6 \\
(-)\end{array}$ & $\begin{array}{l}0.2 \\
(-)\end{array}$ & $\begin{array}{l}3.0 \\
(-)\end{array}$ & $\begin{array}{c}5.88 \\
(-)\end{array}$ & $\begin{array}{c}3.85 \\
(-)\end{array}$ & $\begin{array}{c}14.28 \\
(-)\end{array}$ & $\begin{array}{c}24.01 \\
(-)\end{array}$ & & \\
\hline $\begin{array}{l}\text { Cyperus } \\
\text { exceltatum }\end{array}$ & $\begin{array}{c}0.6 \\
(0.4)\end{array}$ & $\begin{array}{c}0.6 \\
(0.2)\end{array}$ & $\begin{array}{l}1.0 \\
(2.0\end{array}$ & $\begin{array}{c}5.88 \\
(8.33)\end{array}$ & $\begin{array}{l}11.54 \\
(7.69)\end{array}$ & $\begin{array}{c}4.76 \\
(14.81)\end{array}$ & $\begin{array}{c}22.18 \\
(30.83)\end{array}$ & & \\
\hline C. rotundus & 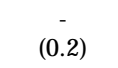 & $\begin{array}{c}- \\
(0.2)\end{array}$ & $\overline{-}$ & $(4.16)$ & $(7.69)$ & $(7.41)$ & $\begin{array}{c}- \\
(19.26)\end{array}$ & & \\
\hline $\begin{array}{l}\text { Eclipta } \\
\text { alba }\end{array}$ & $(0.4)$ & $(0.2)$ & $\begin{array}{c}- \\
(1.0)\end{array}$ & $(8.33)$ & $\begin{array}{c}- \\
(7.69)\end{array}$ & $\begin{array}{c}- \\
(7.41)\end{array}$ & $(19.26)$ & & \\
\hline $\begin{array}{l}\text { Echinochloa } \\
\text { colonum }\end{array}$ & $\begin{array}{l}1.0 \\
(-)\end{array}$ & $\begin{array}{l}0.4 \\
(-)\end{array}$ & $\begin{array}{l}2.5 \\
(-)\end{array}$ & $\begin{array}{l}9.8 \\
(-)\end{array}$ & $\begin{array}{c}7.69 \\
(-)\end{array}$ & $\begin{array}{c}11.9 \\
(-)\end{array}$ & $\begin{array}{l}29.39 \\
(-)\end{array}$ & & \\
\hline $\begin{array}{l}\text { Enhydra } \\
\text { flactuans }\end{array}$ & $\begin{array}{l}0.6 \\
(-)\end{array}$ & $\begin{array}{l}0.4 \\
(-)\end{array}$ & $\begin{array}{l}1.5 \\
(-)\end{array}$ & $\begin{array}{c}5.88 \\
(-)\end{array}$ & $\begin{array}{c}7.69 \\
(-)\end{array}$ & $\begin{array}{c}7.14 \\
(-)\end{array}$ & $\begin{array}{c}20.71 \\
(-)\end{array}$ & & \\
\hline $\begin{array}{l}\text { Eriocaulon } \\
\text { setaceum }\end{array}$ & $\begin{array}{c}2.2 \\
(0.8)\end{array}$ & $\begin{array}{c}0.8 \\
(0.4)\end{array}$ & $\begin{array}{l}2.75 \\
(2.0)\end{array}$ & $\begin{array}{c}21.57 \\
(16.66)\end{array}$ & $\begin{array}{c}15.38 \\
(15.38)\end{array}$ & $\begin{array}{c}13.09 \\
(14.81)\end{array}$ & $\begin{array}{c}50.04 \\
(46.85)\end{array}$ & & \\
\hline Fimbristilis sp. & $(0.6)$ & $\begin{array}{c}- \\
(0.4)\end{array}$ & $(1.5)$ & $(12.5)$ & $\begin{array}{c}- \\
(15.38)\end{array}$ & $(11.11)$ & $(38.99)$ & & \\
\hline $\begin{array}{l}\text { Hediotis } \\
\text { corymbosa }\end{array}$ & $\begin{array}{l}0.4 \\
(-)\end{array}$ & $\begin{array}{l}0.2 \\
(-)\end{array}$ & $\begin{array}{l}2.0 \\
(-)\end{array}$ & $\begin{array}{c}3.92 \\
(-)\end{array}$ & $\begin{array}{c}3.85 \\
(-)\end{array}$ & $\begin{array}{c}9.52 \\
(-)\end{array}$ & $\begin{array}{c}17.29 \\
(-)\end{array}$ & & \\
\hline $\begin{array}{l}\text { Hydrocotyle } \\
\text { rotundifolia }\end{array}$ & $\begin{array}{c}0.6 \\
(0.4)\end{array}$ & $\begin{array}{c}0.6 \\
(0.4)\end{array}$ & $\begin{array}{c}1.0 \\
(1.0)\end{array}$ & $\begin{array}{c}5.88 \\
(8.33)\end{array}$ & $\begin{array}{c}11.54 \\
(15.38)\end{array}$ & $\begin{array}{c}4.76 \\
(7.41)\end{array}$ & $\begin{array}{c}22.18 \\
(31.12)\end{array}$ & & \\
\hline $\begin{array}{l}\text { Lindernia } \\
\text { antipoda }\end{array}$ & $\begin{array}{c}2.2 \\
(1.4)\end{array}$ & $\begin{array}{c}0.8 \\
(0.4)\end{array}$ & $\begin{array}{l}2.75 \\
(3.5)\end{array}$ & $\begin{array}{c}21.57 \\
(29.16)\end{array}$ & $\begin{array}{c}15.38 \\
(15.38)\end{array}$ & $\begin{array}{c}13.09 \\
(25.92)\end{array}$ & $\begin{array}{c}50.04 \\
(70.46)\end{array}$ & & \\
\hline $\begin{array}{l}\text { Lippia } \\
\text { nodiflora }\end{array}$ & $\begin{array}{l}0.6 \\
(-)\end{array}$ & $\begin{array}{l}0.4 \\
(-)\end{array}$ & $\begin{array}{l}1.5 \\
(-)\end{array}$ & $\begin{array}{c}5.88 \\
(-)\end{array}$ & $\begin{array}{c}7.69 \\
(-)\end{array}$ & $\begin{array}{c}7.14 \\
(-)\end{array}$ & $\begin{array}{l}20.71 \\
(-)\end{array}$ & & \\
\hline Panicum sp. & $\begin{array}{c}1.2 \\
(0.6)\end{array}$ & $\begin{array}{c}0.6 \\
(0.4)\end{array}$ & $\begin{array}{c}2.0 \\
(1.5)\end{array}$ & $\begin{array}{l}11.76 \\
(12.5)\end{array}$ & $\begin{array}{c}11.54 \\
(15.38)\end{array}$ & $\begin{array}{c}9.52 \\
(11.11)\end{array}$ & $\begin{array}{c}32.82 \\
(38.99)\end{array}$ & & \\
\hline
\end{tabular}

Data in the parentheses indicate the values of arsenic contaminated irrigated plot. $n=5$.

\section{References}

1. British Geological Survey (BGS) 1999. Groundwater studies for arsenic contamination in Bangladesh. Vol. 1. Mott MacDonald Ltd., United Kingdom.

2. Anderson AC, AA Abdelghani and D McDonell 1980. Screening of four vascular plants for uptake of monosodium methane arsenate (MSMA). Sci. Total Environ. 16: 95-98.

3. Aziz A 2000. Removal of arsenic from water by Azolla and Spirodella. J. Asiat. Soc. Bangladesh (Sci.) 26(2): 271-276. 
4. Jain SK, P Vasudevan and NK Jha 1989. Removal of some heavy metals from polluted water by aquatic plants: Studies on duckweed and water velvet. Biol. Wastes 28: 115126.

5. Wauchope RD 1983. Uptake, translocation and phytotoxicity of arsenic in plants. In: Arsenic: industrial, biomedical and environmental perspectives. (WH Lederer and RJ Fensterheim Eds.), pp. 348-377. Van Nostrand Reinhold, NY.

6. Walsh LM and DR Keeney 1975. Behaviour and phytotoxicity of inorganic arsenicals in soil. In: Arsenical pesticides (EA Woolson Ed.), pp. 35-52. ACS, Washington DC.

7. Zingaro RA and NR Bottino 1983. Biochemistry of arsenic: recent developments. In: Arsenic: industrial, biomedical and environmental perspectives. (WH Lederer and RJ Fensterheim Eds.), pp. 327-347. Van Nostrand Reinhold, NY.

8. Aziz A, S Mohammad Ullah and M Rafique Ullah, M Alam, MA Bashar and Akhtarunnesa Chowdhury 2004. Mitigation of arsenic poisoning of groundwater by employing a locally innovated bioremediation technique. (Mimeo) Ministry of Science and ICT, GOB, Dhaka. 45 pp.

9. Clapham AH 1932 The form of observational units in quantitative ecology. J. Ecol. 20: 192-197.

10. Weaver JE and FE Clements 1977. Plant Ecology. Tata McGraw-Hill Publ. Co. Ltd., New Delhi. pp. 601.

11. Shannon CD and W Weaver 1949. The mathematical theory of communication. Univ. Illinois Press, Urbanca. pp. 117.

12. Simpson EH 1949. Measurements of diversity. Nature 163: 688.

13. Liebig GF, GR Brandford and AP Vanselow 1959. Effects of arsenic compounds on citrus plants in solution culture. Soil Sci. 88(6): 342-348.

14. Woolson EA, JH Axley and PC Kearney 1971. The chemistry and phytotoxicity of arsenic in soils: I. Contaminated field soils. Soil Sci. Soc. Am. J. 35: 938-943.

15. Chaturvedi I 2006. Effects of arsenic concentrations and forms on growth and arsenic uptake and accumulation by Indian mustard (Brassica juncea L.) genotypes. J. Central European Agric. 7(1): 31-40.

16. Jacobs LW, DR Keeney and LM Walsh 1970. Arsenic residue toxicity to vegetable crops grown on plain field sand. Agron. J. 62: 588-591.

17. Chaney RL 1984. Potential effects of sludge-borne heavy metal and toxic organics on soils, plants, and animals and related regulatory guidelines. In: Proc. Pan American Health Organization Workshop on the international transportation, utilization or disposal of sewage sludge, including recommendations (December 12-15). Final Report PNSP/8501, pp. 1-56. Pan American Health Organization. Washington DC.

(Manuscript received on 14 July, 2009; revised on 29 September, 2009) 